\title{
Health Literacy Impact on National Healthcare Utilization and Expenditure
}

\author{
Rafia S. Rasu ${ }^{1 *}$, Walter Agbor Bawa ${ }^{1}$, Richard Suminski ${ }^{2}$, Kathleen Snella ${ }^{3}$, Bradley Warady $^{4}$
}

\begin{abstract}
Background: Health literacy presents an enormous challenge in the delivery of effective healthcare and quality outcomes. We evaluated the impact of low health literacy (LHL) on healthcare utilization and healthcare expenditure. Methods: Database analysis used Medical Expenditure Panel Survey (MEPS) from 2005-2008 which provides nationally representative estimates of healthcare utilization and expenditure. Health literacy scores (HLSs) were calculated based on a validated, predictive model and were scored according to the National Assessment of Adult Literacy (NAAL). HLS ranged from 0-500. Health literacy level (HLL) and categorized in 2 groups: Below basic or basic (HLS <226) and above basic (HLS 2226). Healthcare utilization expressed as a physician, nonphysician, or emergency room (ER) visits and healthcare spending. Expenditures were adjusted to 2010 rates using the Consumer Price Index (CPI). A $P$ value of 0.05 or less was the criterion for statistical significance in all analyses. Multivariate regression models assessed the impact of the predicted HLLs on outpatient healthcare utilization and expenditures. All analyses were performed with SAS and STATA ${ }^{\circledR} 11.0$ statistical software.

Results: The study evaluated 22599 samples representing 503374648 weighted individuals nationally from 20052008. The cohort had an average age of 49 years and included more females (57\%). Caucasian were the predominant racial ethnic group (83\%) and $37 \%$ of the cohort were from the South region of the United States of America. The proportion of the cohort with basic or below basic health literacy was $22.4 \%$. Annual predicted values of physician visits, nonphysician visits, and ER visits were $6.6,4.8$, and 0.2 , respectively, for basic or below basic compared to 4.4 , 2.6, and 0.1 for above basic. Predicted values of office and ER visits expenditures were $\$ 1284$ and $\$ 151$, respectively, for basic or below basic and $\$ 719$ and $\$ 100$ for above basic $(P<.05)$. The extrapolated national estimates show that the annual costs for prescription alone for adults with LHL possibly associated with basic and below basic health literacy could potentially reach about $\$ 172$ billion.

Conclusion: Health literacy is inversely associated with healthcare utilization and expenditure. Individuals with below basic or basic HLL have greater healthcare utilization and expendituresspending more on prescriptions compared to individuals with above basic HLL. Public health strategies promoting appropriate education among individuals with LHL may help to improve health outcomes and reduce unnecessary healthcare visits and costs.

Key words: Health Literacy, Healthcare Expenditure, Utilization, Prescription Expenditure

Copyright: (C) 2015 by Kerman University of Medical Sciences

Citation: Rasu RS, Bawal WA, Suminski R, Snella K, Warady B. Health literacy impact on national healthcare utilization and expenditure. Int J Health Policy Manag. 2015;4(11):747-755. doi:10.15171/ijhpm.2015.151
\end{abstract}

Article History:

Received: 26 November 2014 Accepted: 10 August 2015 ePublished: 17 August 2015

*Correspondence to: Rafia S. Rasu

Email: rrasu2@kumc.edu

\section{Key Messages}

Implications for policy makers

- Health literacy presents an enormous challenge in the delivery of effective healthcare and quality outcomes.

- We found that individuals with below basic or basic compared to above basic health literacy incurred more healthcare visits, have greater total healthcare expenditures.

- Adults with basic or below basic health literacy incurred greater costs for prescription (\$3362) than adults with above basic health literacy level (HLL) (\$910) $(P<.05)$. Public health strategies promoting appropriate education among consumers with low health literacy (LHL) may help to improve health outcomes and reduce unnecessary healthcare visits and costs.

- Health literacy driven health communication strategies may encourage appropriate use of healthcare resources and optimal use of medications.

Implications for public

Health literacy is defined as "the degree to which an individual has the capacity to obtain, communicate, process, and understand basic health information and services to make appropriate health decisions." Health literacy presents a huge challenge in the delivery of effective healthcare and quality outcomes. We found that consumers with low health literacy (LHL) have greater total healthcare visits, expenditures, spending more on prescription medications. The annual cost of LHL for prescriptions alone was $\sim \$ 92$ billion in the United States. Based on this evidence, we suggest health literacy driven health communication strategies may help patients improve their health outcomes, and reduce unnecessary healthcare visits and costs. 


\section{Background}

Health literacy is an important aspect of patient care. Health literacy is defined as "the degree to which an individual can obtain, communicate, process, and understand basic health information and services to make proper health decisions." $1-4$ Only one in 10 adults in the United States are health literate. ${ }^{5}$ Results from the 2003 National Assessment of Adult Literacy (NAAL) ${ }^{6}$ shows that $12 \%$ of US adults were proficient in health literacy level (HLL), 53\% and 22\% reported having had intermediate and basic health literacy, and 14\% had below basic health literacy. ${ }^{3,6}$

Multiple national organizations and institutions, including the Department of Health and Human Services (HHS), ${ }^{3}$ the Joint Commission on the Accreditation of Healthcare Organizations (JCAHO), ${ }^{7}$ and the American Medical Association (AMA) ${ }^{8}$ have prioritized health literacy as a key patient safety and quality of care issue. New federal policy initiatives, including Affordable Care Act (ACA) of 2010, HHS's National Action Plan to Improve Health Literacy, and the Plain Writing Act of 2010 have brought health literacy to a tipping point. $^{3}$

Several studies have reported that HLLs may impact healthcare outcomes including mortality, decreased health status in elderly individuals, as reflected by increased hospitalizations, decreased use of preventative services (mammography and influenza vaccination), increased difficulties taking medications, and interpreting medication labels and health information. ${ }^{4,9,10}$

In 2011, US healthcare expenditures reached $\$ 2.7$ trillion dollars (\$8680/person). ${ }^{11}$ The relationship between health literacy, healthcare utilization and associated expenditures is of particular importance for the US economy. Studies ${ }^{12-16}$ on this issue have typically used validated scales to directly measure health literacy for individuals rather than assess community-level indicators of health literacy. Examples of direct methods include the Rapid Estimate of Adults' Literacy, the Test of Functional Health Literacy in Adults, and the Newest Vital Sign. ${ }^{17-20}$ Using these direct methods on small samples of individuals may limit generalizability to larger scales (eg, state or national level). Information obtained with these methods cannot be used with available census data such as the Medical Expenditure Panel Survey (MEPS). ${ }^{21}$

In contrast, in 2003, the National Center for Education Statistics (NCES) included a health literacy measure within the NAAL, ${ }^{6}$ leading to the first large-scale national assessment of health literacy. Vernon et $\mathrm{al}^{22}$ used this data to approximate the effect of low health literacy (LHL) on healthcare expenditures based on the percentage of individuals reported in the NAAL survey with below basic or basic HLLs (36\%). ${ }^{6}$ They found that one-half of the healthcare costs for individuals with LHL were due to literacy effects resulting in an additional $\$ 237$ billion in healthcare costs. ${ }^{22}$ Although these results are informative, individual data from the MEPS Household Component (MEPS-HC) was not available at that time, therefore, only aggregate data were used to estimate healthcare costs. ${ }^{22}$ This aggregation approach limits the application of their findings, and as a result, maintain a gap in the literature regarding the impact of health literacy on health utilization and expenditures, especially where methods of assessing individual health literacy via census data such as that found in the MEPS-HC are concerned. Therefore, we conducted the current study using MEPS data to examine the impact of health literacy on current national health utilization and expenditure. Specifically, we explored the association between healthcare utilization (office visits-physician or nonphysician, ER visits), prescription expenditures and HLLs. In addition, we extrapolated national data to estimate the national healthcare costs due to LHL within the United States.

\section{Methods}

Study Design and Data Sources

The data source is the MEPS, which are surveys of families and individuals, their medical providers (doctors, hospitals, pharmacies, etc.), and employers in the United States. MEPS ask about the details of health services that Americans use, how often they use them, the cost of these services, and how it is paid for, as well as data on the cost and extent of health insurance available to the US working population. Currently, there are 2 key components of MEPS: the MEPS$\mathrm{HC}$ and the MEPS Insurance Component (MEPS-IC). The MEPS-HC provides data from individual households and the MEPS-IC is an independent survey of employers, providing data on employer-based health insurance. Therefore, in this retrospective database analysis we used the MEPSHC. Specifically, we used the consolidated full year and prescribed medicine event (PME) files of MEPS-HC. The MEPS-HC full year files provide nationally representative estimates of healthcare utilization and expenditures, sources of payment, health insurance coverage, and health status for the US civilian noninstitutionalized population. ${ }^{21}$ The MEPS$\mathrm{HC}$ consolidated full year files also capture information on individual-level health status, use of medical care, medical care expenditures, socio-economic characteristics, and health insurance coverage. The sampling frame was taken from respondents to the National Health Interview Survey (NHIS), performed by Agency for Healthcare Research and Quality (AHRQ). The MEPS-HC full-year consolidated data files were from 2005 to 2008 (MEPS HC-106 Panel 10 2005-2006; MEPS HC-114 Panel 11 2006-2007; MEPS HC-122 Panel 12 2007-2008). The MEPS-HC collects data from nationally representative sample households via an overlapping panel design. This overlapping collection method provides continuous estimates of healthcare expenditures for 2 panels each calendar year.

The MEPS-HC PME files from the same period were used to estimate prescribed medicine utilization and expenditures for 2005-2008. ${ }^{23,24}$ The MEPS-HC PME files provide detailed information on household-reported prescribed medicines. Each record on this event file represents a unique PMEs. ${ }^{21}$ Detailed information on these variables can be found at MEPS website. ${ }^{21}$ The study used this event file to calculate the total prescription expenditure for the period 2005-2008, grouped by household respondent. ${ }^{23,24}$

Data from MEPS-HC PME files were merged with MEPS$\mathrm{HC}$ consolidated full year files using the respondent unique identifier and the panel number to obtain the final analytical dataset. The longitudinal weights given in the MEPS-HC consolidated full year files were used to derive national estimates. We defined the study inclusion criteria as follows: 
(a) individuals aged $\geq 18$; $(b)$ participated in all five interview rounds in a set calendar year; and $(c)$ individuals with or without events in the PME file. The 2005-2008 MEPS included 22599 participants who met the inclusion criteria, corresponding to a weighted national estimate of 503374648 persons from 2005-2008.

\section{Predictive Model for Health Literacy Assessment}

This study used a validated, predictive model that defined health literacy using patient demographic and socioeconomic factors from US census data. The model was described by Martin et $\mathrm{al}^{25}$ in 2009 and was based on the health literacy scores (HLS) presented in the 2003 NCES NAAL report. ${ }^{6}$ The NAAL evaluated health literacy on a 0 to 500 point scale $($ mean $=245$, standard deviation $=55) .{ }^{26}$ The National Research Council categorized HLS into 4 ordinal categories to reflect an individual's ability to successfully finish tasks of a given complexity: below basic (0-184), basic (185-225), intermediate (226-309), and proficient (310-500). Below basic health literacy skills indicate the ability only to perform tasks such as circling the date on an appointment slip. Basic health literacy skills indicate the ability, for example, to give 2 reasons why it is necessary for a person to be tested for a specific disease, using evidence from a clearly written pamphlet. Intermediate health literacy individuals can perform moderately demanding health literacy activities, such as, establishing at what time a person can take a prescribed medication from the prescribed drug label directions. Individuals with proficient health literacy skills are able to perform more complex and challenging literacy activities, for example, calculating their share of employer health costs from a table.

The multivariate model presented by Martin et $\mathrm{al}^{25}$ predict health literacy as a continuous outcome HLS. The HLS was then used to create 2 categories: below basic or basic (HLS $<226$ ) and above basic (HLS $\geq 226$ ).

We applied the predictive model on the MEPS-HC to derive a proxy for HLS, which ranged from 0-500; matching the literacy scale from the 2003 NAAL report. ${ }^{6,25}$ Two HLLs were also created by categorizing HLS as below basic or basic (HLS $<226$; including below basic HLS: 0-184 and basic HLS: 185225) and above basic (HLS $\geq 226$; including intermediate HLS: 226-309 and proficient HLS: 310-500).

In order to apply the predictive model on MEPS-HC, we used unstandardized regression coefficients presented in Martin et $\mathrm{al}^{25}$ in 2009 to reconstruct the multivariate model with known variables from MEPS-HC data. The following variables were extracted from the analytical dataset (a merger of MEPS-HC consolidated full year files and MEPS-HC PME files): agecategorized as $18-24,25-39,40-49,50-59,60-74$, or $\geq 75$ years; gender-categorized as male or female; race-categorized as white, black, American Indian/Alaska Native, Asian, Native Hawaiian/Pacific Islander and Multiracial; ethnicity: Hispanic and Non-Hispanic; income-categorized as poor, near poor, or low income ( $\leq 2.0$ times poverty level), middle income (2.1-4 times poverty level), or high income $(>4.0$ times poverty level); reside in a metropolitan area (MSA) or a non-metropolitan area (non-MSA); individual's educational attainment - categorized as 'still in high school', 'still in college/some college,' 'less than high school,' 'high school or equivalent,' and 'bachelor's degrees or higher'; marital status categorized as married, divorced, widowed/separated, or never married; and language spoken at home - categorized as English or other. All the above variables were used in the predictive model presented by Martin et $\mathrm{al}^{25}$ in addition to the 'time in the United States' variable which was not available in MEPS-HC. All variables, with the exception of living in a MSA and language spoken at home made significant independent contributions to the predictive model. The adjusted $\mathrm{R}^{2}$ for the multivariate regression model indicates that the demographic predictors accounted for $30 \%$ of the variance in HLSs. The absence of the 'Time in the United States' variable in MEPS$\mathrm{HC}$ can be seen as one of the limitations in our reconstructed model since we will be assuming that every respondent was born in the United States.

\section{Statistical Analysis}

This study examined the impact of predicted HLLs on outpatient healthcare utilization and expenditures. HLL was created from HLS categorized in 2 groups: (1) Below basic or basic (HLS <226) and (2) above basic (HLS $\geq 226$ ). Healthcare utilization and expenditures are the dependent variables. Healthcare utilization is expressed as a physician visit, nonphysician visit, or ER visits. Physician visits are performed by a physician, while nonphysician visits are performed by physician assistants (PAs), advance practice nurses (APNs or ARNPs), or chiropractors. Healthcare expenditures are expressed as visits and as prescription expenditures. The estimates of annual visits and expenditures are calculated by averaging the expenditures and visits over the three calendar years' data.

We used multivariate regression models to assess the relationship between the dependent variables: (1) physician visits; (2) nonphysician visits; (3) ER visits; (4) office-based visit expenditures (OFVisitsEXP); (5) ER visit expenditures; (6) prescription medication expenditures (RxEXP) and the independent variable HLL. HLS and hence HLL were constructed using socio-economic and demographic variables from MEPS-HC survey and thus including it directly as a key explanatory variable in multivariate regression models will make the HLL variable endogenous resulting from uncontrolled confounders especially from the 'age' and 'education' variables. In order to account for endogeneity of HLL and effectively control confounders, we used the 2-stage residual inclusion (2SRI) technique. The first-stage regression involved estimates of HLL using instrumental or possible confounders (age; gender; ethnicity; income; reside in a MSA or a non-MSA; individual's educational attainment; marital status; and language spoken at home). The goal of this first stage regression is to obtain a predicted residual term which will be included as an additional regressor in the 2-stage multivariate model. We choose the 2SRI method to handle the endogeneity and confounding issues as compared to the 2-stage prediction substitution (2SPS) method because the 2SRI shows more consistent results and simulation studies recommend against 2 SPS and favor 2 SRI. ${ }^{27}$ The 2 -stage multivariate regression models generate 6 different models with 6 different outcome measures to calculate utilization and expenditures: (1) physician visits; (2) nonphysician visits; (3) ER visits; (4) office-based visit expenditures (OFVisitsEXP); 
(5) ER visit expenditures (ERvisitsEXP); (6) prescription medication expenditures (RxEXP). Each of these measures was used in a multivariate regression with HLL as independent variable and covariates: age, gender, ethnicity, income, reside in a MSA or a non-metropolitan area non-MSA, individual's educational attainment, marital status, and language spoken at home, and the residual term (from first stage regression). Because the variables for the number of visits can assume small (non-negative integer values), we used negative binomial regression to model these variables. ${ }^{28}$ Emergency room (ER) visit and prescription medication expenditures were modeled using Poisson family generalized linear regression (GLM) with log link function. All other expenditure data were modeled using the gamma family GLM with log link function. Box-Cox and Park tests were used to determine model family and link function, respectively. All statistical analyses were weighted using longitudinal weights.

The regression models examined associations between each outcome variable (physician visits, nonphysician visits, ER visits, OFVisitsEXP, ERvisitsEXP or RxEXP) and HLLs. The model was later adjusted for other variables they were insurance coverage (categorized as no insurance, private insurance, or public insurance, eg, Medicaid, Medicare); and region (categorized as South, West, Northeast, or Midwest). The estimation of annual expenditures was calculated by averaging the expenditures and visits over the three calendar years data was collected. In a post hoc analysis, the differences in annual adjusted expenditures were estimated for the study population and extrapolated nationally using 2010 US census data $(\mathrm{N}=308745538) .{ }^{29}$ To account for inflation and to match the 2010 census year, expenditures were adjusted to 2010 rates using the Consumer Price Index. ${ }^{26}$ A $P$ value of 0.05 or less was considered for statistical significance. All analyses were performed with SAS and STATA ${ }^{\circledR} 11.0$ statistical software.

\section{Results}

\section{Descriptive Statistics}

The study included a total of 22599 samples representing 503374648 weighted individuals nationally from 2005-2008 (Table 1). The cohort had an average age of 49 years (standard deviation $[\mathrm{SD}] \pm 17.8)$ and included more females $(57 \%)$. Caucasian were the predominant racial ethnic group (83\%) and $37 \%$ of the cohort were from the South region of the United States of America. Overall, the mean HLS was 247.5 $(\mathrm{SD} \pm 28.3)$. The HLS statistical mean varied by individual demographic and socio-economic characteristics such as age, gender, marital status, region, and poverty levels (Table 1). Approximately $22 \%$ of individuals have below basic or basic HLL and about $2 \%$ of these individuals had below basic HLS. None of these individuals had a proficient HLL, therefore, this level was excluded from all tables and figures.

\section{Healthcare Utilization}

On average the cohort had 4.9 ( $\mathrm{SD} \pm 6.8)$ physician visits, 3.0 $(\mathrm{SD} \pm 3.9)$ nonphysician visits, and $0.1(\mathrm{SD} \pm 0.3)$ ER visits (Table 1). Adults with below basic or basic health literacy had relatively more annual mean standard error ( \pm standard error [SE]) physician visits: $5.8 \pm 0.1$ vs. $4.5 \pm 0.1$, nonphysician visits: $4.1 \pm 0.1$ vs. $2.7 \pm 0.0$, ER visits: $0.2 \pm 0.0$ vs. $0.1 \pm 0.0$ (Figure 1) compared to adults with above basic HLL. For all visit types, those with below basic health literacy incurred the most visits (Figure 1). The unadjusted analyses (Table 2) show a significant impact of health literacy on office and ER visits. Compared to individuals with above basic health literacy, those with below basic or basic health literacy averaged more physician $(+2.9)$, nonphysician $(+2.8)$, and ER $(+0.2)$ visits annually. The adjusted analyses (Table 2 ) confirmed the trend that individuals with below basic or basic health literacy had more total physician or nonphysician visits, incurred more visits than individuals with above basic health literacy. For instance, after adjusting for other variables (individuals insurance types and regions) (Table 2), individuals with below basic or basic health literacy averaged 6.6 total physician office visits, 4.8 nonphysician visits, and $0.2 \mathrm{ER}$ visits annually, compared with 4.4 total physician visits, 2.6 nonphysician visits, and 0.1 ER visits for those with above basic health literacy $(P<.05$ for both comparisons).

\section{Healthcare Expenditure}

The MEPS-HS annual total visit expenditure mean was $\$ 926 /$ person (95\% CI: 894.6-957.7) and the average total annual amount spent on prescription medications was \$1214 (95\% CI: 1171.9-1256.4) (Table 1). Health illiterate individuals spent more on prescription drugs: \$1862, 95\% CI: 1776-1948 vs. \$1027, 95\% CI: 981-1074. Prescription medications were the highest expenditure for individuals with below basic or basic health literacy (Figure 2). The unadjusted analyses (Table 2) show a significant impact of health literacy on expenditures for office and ER visits, and prescriptions. Those with below basic or basic health literacy experienced higher expenses for office visits $(+\$ 556)$, ER visits $(+\$ 52.2)$, and prescriptions (+\$2939) (Table 2). The adjusted analyses (Table 2) confirmed the trend that individuals with below basic or basic health literacy incurred more visit and prescription medications expenditures than individuals with above basic health literacy. For instance, after adjusting for other variables (individuals insurance types and regions) (Table 2), below basic or basic health literacy individuals averaged $\$ 1284$ office, \$151 ER visits, and \$3362 prescriptions expenditures annually, compared with $\$ 719$ office, $\$ 100$ ER visits, and $\$ 910$ in those with above basic health literacy $(P<.05$ for both comparisons). Prescription expenditures were significantly greater in the below basic group (\$3362) compared to the above basic group $(\$ 910)(P<.05$; Table 2$)$.

Figure 3 depicted the annual costs for ER visits ( $\$ 1.9$ billion), total office visits ( $\$ 21.2$ billion) and prescription medications ( $\$ 92.2$ billion) attributed to LHL. These extrapolated national estimates showed that the annual costs possibly associated with LHL were $\$ 215.1$ billion (Figure 3 ). This increased healthcare cost associated with LHL was not equally divided between the three expenses. In fact, $80 \%$ of the overall increased costs were from prescription medications followed by office visits (18.4\%) and ER (1.6\%) visits.

\section{Discussion}

Previous studies ${ }^{12-16}$ examining associations between healthcare utilization, expenditures and health literacy were limited to individual-level interpretations given their measurement approaches. ${ }^{12-16}$ To address this shortcoming, we conducted the current study, which is multilevel (eg, 
Table 1. Descriptive Statistics for Cross-Sectional Patient Study Population ( $n=22599$, Weighted Population $=503$ million) From 2005 to 2008

\begin{tabular}{|c|c|c|c|c|c|}
\hline \multirow{2}{*}{ Variable } & \multirow{2}{*}{ Percent } & \multicolumn{2}{|c|}{ Health Literacy } & \multirow{2}{*}{ Mean Total Visits } & \multirow{2}{*}{ Mean Total Expenditure } \\
\hline & & Mean Literacy Score ${ }^{a}$ & \% Above Basic (HLS $\geq 226$ ) & & \\
\hline All participants (Mean \pm SD) & 100 & $247.5 \pm 28.3$ & 77.6 & $7.9 \pm 10.2^{b}$ & $\$ 2140^{c}$ \\
\hline \multicolumn{6}{|l|}{ Gender } \\
\hline Male $^{\ddagger}$ & 43.4 & 244.5 & 76.8 & 6.8 & 2008.7 \\
\hline Female & 56.6 & 250.2 & 78.2 & 8.8 & 2241.3 \\
\hline \multicolumn{6}{|l|}{ Age, y (Mean \pm SD: $48.7 \pm 17.8$ ) } \\
\hline $18-24^{\neq}$ & 9.9 & 258.1 & 94.1 & 4.2 & 774.9 \\
\hline $25-39$ & 24.0 & 264.5 & 92.5 & 5.9 & 1194.0 \\
\hline $40-49$ & 18.5 & 253.1 & 88.3 & 6.4 & 1661.8 \\
\hline $50-64$ & 27.3 & 248.7 & 85.7 & 8.9 & 2804.6 \\
\hline $65-74$ & 10.5 & 223.6 & 39.9 & 11.4 & 3407.7 \\
\hline $75+$ & 9.8 & 209.5 & 22.5 & 13.3 & 3518.8 \\
\hline \multicolumn{6}{|l|}{ Race } \\
\hline White ${ }^{\ddagger}$ & 83.4 & 252.5 & 84.3 & 8.2 & 2183.7 \\
\hline American Indian/Alaska Native & 0.8 & 225.2 & 52.3 & 7.4 & 2232.3 \\
\hline Asian & 3.6 & 244.6 & 75.1 & 5.7 & 1417.7 \\
\hline Hawaiian/Pacific Islander & 0.3 & 240.7 & 79.0 & 5.7 & 2476.5 \\
\hline Multiracial & 1.3 & 231.7 & 63.8 & 8.5 & 2441.7 \\
\hline \multicolumn{6}{|l|}{ Ethnicity } \\
\hline Hispanic ${ }^{\ddagger}$ & 10.5 & 246.9 & 83.7 & 6.2 & 1533.2 \\
\hline Non-Hispanic & 89.5 & $247.8^{d}$ & 76.9 & 8.1 & 2211.7 \\
\hline \multicolumn{6}{|l|}{ Insurance } \\
\hline Any private ${ }^{\ddagger}$ & 73.5 & 253.7 & 84.3 & 7.7 & 2077.0 \\
\hline Public only & 16.8 & 223.2 & 45.1 & 11.0 & 3106.5 \\
\hline Uninsured & 9.7 & 245.2 & 83.3 & 4.3 & 943.1 \\
\hline \multicolumn{6}{|l|}{ Education level } \\
\hline Still in $\mathrm{HS}^{\neq}$ & 10.7 & 227.8 & 56.0 & 7.0 & 1906.4 \\
\hline Still in college/some college & 1.4 & 263.4 & 99.3 & 4.5 & 923.1 \\
\hline >Bachelors & 35.7 & 273.1 & 99.0 & 8.5 & 2143.9 \\
\hline \multicolumn{6}{|l|}{ Poverty level } \\
\hline Poor/negative ${ }^{\ddagger}$ & 10.3 & 228.5 & 58.0 & 8.5 & 2266.0 \\
\hline Near Poor & 4.2 & 224.4 & 48.9 & 8.9 & 2498.4 \\
\hline Low income & 12.4 & 233.4 & 62.4 & 7.8 & 2291.5 \\
\hline Middle income & 29.9 & 244.8 & 76.0 & 7.5 & 2023.6 \\
\hline High income & 43.3 & 260.7 & 90.5 & 8.1 & 2113.1 \\
\hline \multicolumn{6}{|l|}{ Marital status } \\
\hline Married $^{\ddagger}$ & 56.8 & 252 & 82.5 & 8.0 & 2184.4 \\
\hline Divorced, widowed/separated & 21.7 & 231.4 & 58.0 & 9.6 & 2724.4 \\
\hline Never married & 21.4 & $252.8^{d}$ & 84.5 & 6.1 & 1431.9 \\
\hline \multicolumn{6}{|l|}{ Language in home } \\
\hline English $^{*}$ & 91.8 & 248.1 & 77.6 & 8.1 & 2211.5 \\
\hline Others & 8.2 & 243.6 & 78.7 & 5.7 & 1347.3 \\
\hline \multicolumn{6}{|l|}{ Region } \\
\hline Northeast ${ }^{\ddagger}$ & 18.8 & 248.6 & 77.7 & 9.1 & 2190.7 \\
\hline Midwest & 22.9 & $248.9^{d}$ & 79.5 & 7.9 & 2289.6 \\
\hline South & 36.8 & 245.1 & 74.1 & 7.6 & 2095.5 \\
\hline West & 21.6 & 250.3 & 81.6 & 7.6 & 2014.5 \\
\hline \multicolumn{6}{|l|}{ Rurality } \\
\hline Non-MSA & 17.2 & 242.1 & 74.3 & 7.4 & 2258.0 \\
\hline MSA & 82.8 & 248.9 & 78.3 & 8.1 & 2115.9 \\
\hline \multicolumn{6}{|l|}{ Health literacy } \\
\hline Below basic HLS (0-184) & 1.5 & 171.8 & $\mathrm{n} / \mathrm{a}$ & 10.3 & 3398.0 \\
\hline Basic HLS (185-225) & 20.9 & 209.8 & $\mathrm{n} / \mathrm{a}$ & 10.1 & 2949.0 \\
\hline Intermediate HLS (226-309) & 77.6 & 259.4 & 100.0 & 7.3 & 1899.0 \\
\hline
\end{tabular}

Abbreviations: HLS, health literacy score; MSA, metropolitan area; HS, high school; $n / a$, not available.

${ }^{a}$ HLS by National Assessment of Adult Literacy (NAAL): 0-184 (below basic), 185-225 (basic), 226-309 (intermediate), 310-500 (proficient).

${ }^{\mathrm{b}}$ Mean total visits (mean physician-visits [4.8 \pm 6.8 ]; nonphysician visits [3.0 \pm 3.9$]$; ER visits [0.2 \pm 0.3$]$ )

${ }^{c}$ Mean total expenditure (mean visits expenditure ( $\left.\$ 926,95 \% \mathrm{Cl}=894.6-957.7\right)$; $\mathrm{Rx}$ expenditure $(\$ 1214,95 \% \mathrm{Cl}=1171.9-1256.4)$.

${ }^{\mathrm{d}}$ Non-significant difference $(P>.05)$ in mean HLS between the specified group and the comparison category (as denoted by $\left.¥\right)$.

For all other mean HLS, $P<.05$ between the specified group and the comparison category (as denoted by $\ddagger$ ).

All values are annualized. 


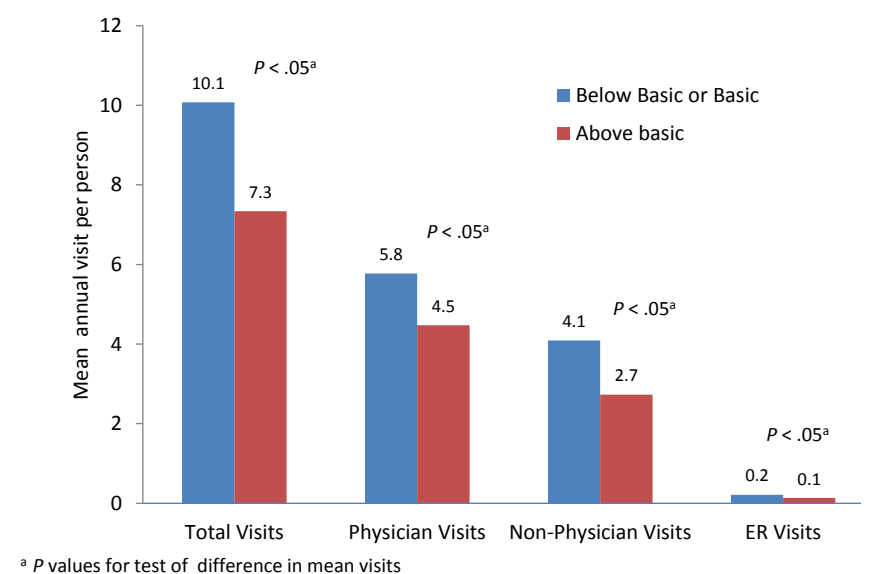

Figure 1. Mean Number of Visits Per Person Based on Health Literacy Level (HLL).

household and individual) connects healthcare utilization and expenditure data derived from a national, standardized survey of US adults (MEPS-HC) with health literacy.

Only 2 recent studies evaluated healthcare utilizations using the MEPS-HS data. ${ }^{30,31}$ One of them evaluated the influence of family composition on office visits, ER visits, and prescription medication use in a pediatric population (age $\leq 18$ ). The investigators noted significant differences in these parameters between households with three or more children versus one child and between households with multiple adults compared with households with one adult. ${ }^{30}$ Another study evaluated the association between individual demographic factors and healthcare resources (access to physician care and preventative healthcare services) and compared rates between the United States and Canada. ${ }^{31}$ Although some differences between countries were found, authors attributed those to dissimilarities in health insurance structures. ${ }^{31}$ The results of the current study suggest that health literacy may also explain some of the variation in healthcare utilization and expenditures they noted between levels of human aggregation

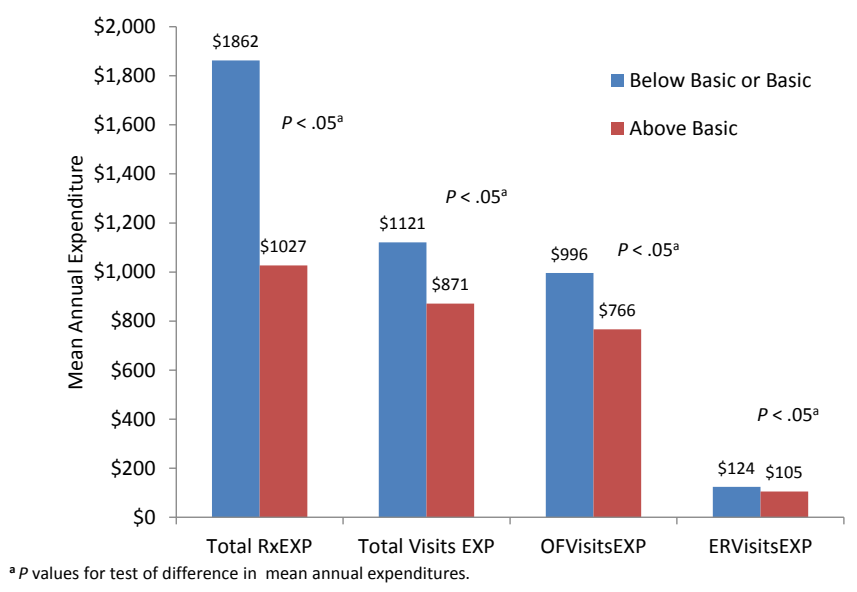

Figure 2. Annual Mean Healthcare Expenditure Per Person by Health Literacy Level (HLL).

(eg, household, country). It will be important for future studies to further describe the relative role of health literacy and to further delineate the extent to which health literacy acts as a determinant of increased healthcare utilization and expenditures.

Health literacy is possibly associated with office visits, visits expenditures, and spending on prescription medications. These effects persisted even after adjusting for other variables including insurance types. Individuals with below basic or basic HLLs had a significantly greater frequency of physician visits, nonphysician visits, and ER visits. Lower health literacy individuals also had greater prescription expenditures compared to individuals with above basic health literacy. Possible explanations for these increased prescriptions costs for those include greater severity of disease (especially in the 65 and above age group which exerted about a 56\% influence on the prescription medication expenditures) and perhaps a greater reliance on prescription therapy in contrast to a greater emphasis on preventative healthcare for those with higher health literacy. A greater emphasis on preventative services

Table 2. Adjusted and Unadjusted Analysis of Health Literacy Level on Number of Visits and Expenditures

\begin{tabular}{lcccccc}
\hline Variable & Physician visits (SE) & Nonphysician visits (SE) & ER Visits (SE) & OFVisitsEXP (SE) & ERVisitsEXP (SE) & RXEXP (SE) \\
\hline Unadjusted & & & & & & \\
$\quad$ Below basic or basic & $7.1(0.2)$ & $5.3(0.2)$ & $0.3(0.01)$ & $\$ 1276(55.4)$ & $\$ 152(11.0)$ & $\$ 3835(185.9)$ \\
Above basic & $4.2(0.1)$ & $2.6(0.0)$ & $0.1(0.0)$ & $\$ 720(16.9)$ & $\$ 99.8(3.5)$ & $\$ 896(22.5)$ \\
$\quad$ Change & $+2.9(0.2)$ & $+2.8(0.1)$ & $+0.2(0.0)$ & $+\$ 556(38.5)$ & $+\$ 52.2(7.5)$ & $2,939(163.4)$ \\
Adjusted & & & & & \\
$\quad$ Below basic or basic & $6.6(0.2)$ & $4.9(0.1)$ & $0.2(0.0)$ & $\$ 1284(62.1)$ & $\$ 151(11.3)$ & $\$ 3362(167.7)$ \\
Above basic & $4.3(0.1)$ & $2.6(0.0)$ & $0.1(0.0)$ & $\$ 719(43.7)$ & $\$ 100(3.4)$ & $\$ 910(22.8)$ \\
$\quad$ Change & $+2.3(0.1)$ & $+2.2(0.1)$ & $+0.1(0.0)$ & $+\$ 565(18.4)$ & $+\$ 51(7.9)$ & $+\$ 2452(144.9)$ \\
\hline
\end{tabular}

Abbreviation: $\mathrm{SE}$, standard error; ER, emergency room.

All values in table are statistically significant with $P<.0001$.

All results are annualized, $n=22433$ (weighted population $=500,367,522$ ). Survey period: 2005-2008: 3 person years.

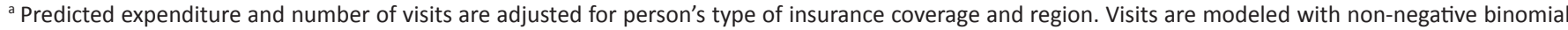
regression. ER expenditure was modeled using Poisson family GLM with log link function. All other expenditure data were modeled using the gamma family GLM with log link function. Box-Cox and Park tests were used to determined model family and link function. Calculation of total cost and Rx expenditure due to low health literacy $(\mathrm{LHL}$ ) as follows: Weighted population $=503374648$; $\%$ LHL in population $=22.4 \%$ (from Table 1); Weighted LHL population $=112755921 ;$ per year $=37585$ 307; Extrapolated visit cost $=[$ change (OFvisitsEXP) + change $($ ERvisitEXP)] $* 37585307=\$ 23152549143$; Extrapolated Rx expenditure $=$ change $($ RxEXP)*37585 $307=\$ 92159172888$; US population (census.gov) $=308745$ 538; Estimated LHL population $=22.4 \% * 308745538=69159001$; Extrapolated visit cost (adjusted for 2010 inflation) $=[$ change (OFvisitsEXP) + change (ERvisitEXP)]* $69159001=\$ 42,601944315$; Extrapolated Rx expenditure = change $($ RxEXP)* $69159001=\$ 169577869255$. 


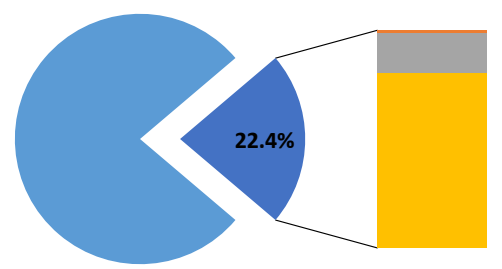

\begin{tabular}{|c|c|c|}
\hline \multicolumn{2}{|c|}{ Above Basic HLL 78\% } & Emergency Room Visit Expenditures 0.4\% \\
\hline Office Visit Expe & Prescription Medicatio & penditures $17.9 \%$ \\
\hline $\begin{array}{l}\text { Study population } \\
\text { (annually) }\end{array}$ & $\begin{array}{l}\text { Percent distribution of increased } \\
\text { expenditures due to low health literacy }\end{array}$ & 2010 Population \\
\hline \$92.2 Billion & $+80 \%$ & $\$ 171.9$ Billion \\
\hline$\$ 21.2$ Billion & $+18.4 \%$ & \$39.6 Billion \\
\hline \$1.9 Billion & $+1.6 \%$ & \$ 3.6 Billion \\
\hline \$115.3 Billion & $100 \%$ & $\$ 215.1$ Billion \\
\hline
\end{tabular}

Low health literacy = basic or below basic HLL. Study population (annually) N = 167791549 . United States population estimates were based on the 2010 US Census. 2010 Population $N=308745$ 538. 2010 expenditures were adjusted for inflation for $\Delta$ office visit (\$573), $\Delta$ emergency room $(\$ 52)$, and $\Delta$ prescription medications (\$2486) using the Consumer Price Index to account for inflation. ${ }^{29}$

Figure 3. Change in Annual Study Population and National United States Population Expenditures Attributed to Low Health Literacy Level (HLL).

and self-management could also explain the relatively lower ER utilization rates in higher health literate individuals. ${ }^{32}$ Another possible explanation for increased prescription expenditures could be that prescriptions are purchased, but are not used optimally by those with LHL thus necessitating more medications to be purchased. This phenomenon also has been observed in several prior studies evaluating LHL and prescription medication use (eg, difficulty in interpreting prescription labels, auxiliary labels, and scheduling medications times throughout the day, etc.).9,33-41 Although pharmacists or healthcare providers can use health literacy assessments to maximize therapy outcomes, interventions by pharmacists to provide literacy-appropriate information and support have met with mixed success. ${ }^{37-39}$ Of note, the MEPS prescription medication expenditures included pharmacy records which tracked both insurance and out-ofpocket expenses. Insurance type was included in the adjusted analysis; therefore, the increased costs are less likely to be due to differences in private, public, or self-insurance coverage. The reasons for the increased expenditures and use of healthcare resources are likely multifactorial. If the increased number of ER visits in below basic individuals is in part due to chronic care or conditions that may be managed in an office setting, transitioning that care to the office could result in more cost effective care. Additional office visits focusing on management or prevention of health conditions may also result in fewer ER visits for exacerbations or preventable conditions. The lower number of ER visits for above basic individuals may reflect increased use of preventative services and the increased use of electronic media to identify and manage symptoms. There is a plethora of literature attesting to the efficacy of chronic disease self-management as a mechanism for significantly reducing healthcare burden and improving health in general. ${ }^{42,43}$

Public health initiatives considering health literacy driven health communication strategies may encourage appropriate use of healthcare resources and optimal use of medications. Recent national initiatives like "Health Literacy Universal Precautions Toolkit" endorsed by AHRQ encourage "taking specific actions that minimize risk for everyone when it is unclear which patients may be affected." Universal precautions on health literacy are needed because "providers do not always know which patients have limited health literacy." In addition, census level data, such as the data used within this study, can help identify areas within the community that may benefit from targeted services. Healthcare entities serving these areas could educate their providers and staff in accessing ${ }^{42}$ health literacy and providing information to patients at the appropriate literacy level ${ }^{44-46}$ which may, in turn, help achieve better health outcomes. ${ }^{3,4}$

Our analysis indicated that overall visits and expenditures were inversely related to health literacy, there is the possibility that changes in observed expenditures may have resulted from sampling variation. However, increased healthcare costs due to LHL were also been found by Vernon et al. ${ }^{22}$ In addition, the NALS database provided evidence that individuals with 'inadequate' functional literacy had increased hospitalization rates, hospitalization days, and physician visits. ${ }^{32}$

Several limitations are worth noting. First, predicted health literacy may not reflect the actual level of health literacy that might be detected by directly measuring health literacy. However, it would be very costly and time consuming to directly access health literacy in a large, nationally representative sample. Therefore, the validated model provided in this study presents a unique opportunity to explore the current health literacy situation nationally. Second, the model derived from NAAL data is limited to the ability to read materials to accomplish health related tasks. Since NAAL assessed health literacy using only printed materials, the model fails to capture a broader 
conceptualization of health literacy. For example, oral language (speaking) or aural language (listening) skills were not included as predictors in the model as they were not available in MEPS dataset. Third, because of the nature of the MEPS dataset, we could not establish causality and could only address the associations between health literacy and health services expenditures and utilization. Although we are using a validated tool to calculate HLL, however; that may not reflect accurate HLL.

\section{Conclusion}

The main finding from this study was that health literacy is associated with healthcare utilization and expenditures. Individuals with below basic or basic compared to above basic health literacy individuals incurred more visits and spent more on visits and prescription medications.

Public health communications promoting health literacy driven appropriate strategies, such as adopting Health Literacy Universal Precautions Toolkit, may help to improve health outcomes and reduce unnecessary healthcare costs.

\section{Ethical issues}

We utilized AHRQ provided MEPS data. Publicly available de-identified data was used in this study.

Competing interests

Authors declare that they have no competing interests.

\section{Authors' contributions}

Concept and design (RSR); acquisition of data (WAB); statistical analysis (WAB); data and result interpretation (RSR and WAB); drafting of the manuscript (RSR, WAB, RS, KS, and BW); critical revision of the manuscript for important content (RSR, WAB, KS, RS, and BW); and supervision (RSR).

\section{Authors' affiliations}

'University of Kansas School of Pharmacy, Lawrence, KS, USA. ${ }^{2}$ Institute for Biobehavioral Health Research, National Development and Research Institutes (NDRI) New York, NY and Leawood, KS, USA. 'Ben and Maytee Fisch College of Pharmacy, University of Texas at Tyler, Tyler, TX, USA. ${ }^{4}$ Children's Mercy Hospital, Kansas City, MO, USA.

\section{References}

1. Selden CR, Zorn M, Ratzan SC, Parker RM, eds. Current Bibliographies in Medicine: Health Literacy. Bethesda, MD: National Institutes of Health, U.S. Department of Health and Human Services; 2000.

2. Centers for Disease Control and Prevention (CDC). Learn About Health Literacy. http://www.cdc.gov/healthliteracy/Learn/index. html. Accessed July 15, 2012.

3. Koh HK, Berwick DM, Clancy CM, et al. New federal policy initiatives to boost health literacy can help the nation move beyond the cycle of costly 'crisis care'. Health Aff (Millwood). 2012;31(2):434-443. doi:10.1377/hlthaff.2011.1169

4. Rudd RE. Improving Americans' health literacy. N Engl J Med. 2010;363(24):2283-2285. doi:10.1056/NEJMp1008755

5. Pei L, Legge D, Stanton P. Policy contradictions limiting hospital performance in China. Policy Stud. 2000;21(2):99-113.

6. Kutner M, Greenberg E, Jin Y, Paulsen C. The Health Literacy of America's Adults: Results From the 2003 National Assessment of Adult Literacy (NCES 2006-483). Washington, DC: US Department of Education; 2006.

7. The Joint Commission. "What did the doctor say?": Improving health literacy to protect patient safety. Oakbrook Terrace, IL:
The Joint Commission; 2007.

8. American Medical Association (AMA). Health literacy. http:// www.ama-assn.org/ama/pub/about-ama/ama-foundation/ our-programs/public-health/health-literacy-program.page? Accessed July 27, 2012. Published 2012.

9. Berkman ND, Sheridan SL, Donahue KE, Halpern DJ, Crotty K. Low health literacy and health outcomes: an updated systematic review. Ann Intern Med. 2011;155(2):97-107. doi:10.1059/00034819-155-2-201107190-00005

10. Mottus R, Johnson W, Murray C, Wolf MS, Starr JM, Deary IJ. Towards Understanding the Links Between Health Literacy and Physical Health. Health Psychol. 2013. doi:10.1037/a0031439

11. Hartman M, Martin $A B$, Benson J, Catlin A. National health spending in 2011: overall growth remains low, but some payers and services show signs of acceleration. Health Aff (Millwood). 2013;32(1):87-99. doi:10.1377/hlthaff.2012.1206

12. Chen JZ, Hsu HC, Tung HJ, Pan LY. Effects of health literacy to self-efficacy and preventive care utilization among older adults. Geriatr Gerontol Int. 2013;13(1):70-76. doi:10.1111/j.14470594.2012.00862.x

13. Cho YI, Lee SY, Arozullah AM, Crittenden KS. Effects of health literacy on health status and health service utilization amongst the elderly. Soc Sci Med. 2008;66(8):1809-1816. doi:10.1016/j. socscimed.2008.01.003

14. Hardie NA, Kyanko K, Busch S, Losasso AT, Levin RA. Health literacy and health care spending and utilization in a consumerdriven health plan. J Health Commun. 2011;16(suppl 3):308321. doi:10.1080/10810730.2011.604703

15. Lincoln A, Paasche-Orlow MK, Cheng DM, et al. Impact of health literacy on depressive symptoms and mental health-related: quality of life among adults with addiction. $J$ Gen Intern Med. 2006;21(8):818-822. doi:10.1111/j.1525-1497.2006.00533.x

16. Welch VL, VanGeest JB, Caskey R. Time, costs, and clinical utilization of screening for health literacy: a case study using the Newest Vital Sign (NVS) instrument. J Am Board Fam Med. 2011;24(3):281-289. doi:10.3122/jabfm.2011.03.100212

17. Davis TC, Crouch MA, Long SW, et al. Rapid assessment of literacy levels of adult primary care patients. Fam Med. 1991;23(6):433-435.

18. Davis TC, Long SW, Jackson RH, et al. Rapid estimate of adult literacy in medicine: a shortened screening instrument. Fam Med. 1993;25(6):391-395.

19. Osborn CY, Weiss BD, Davis TC, et al. Measuring adult literacy in health care: performance of the newest vital sign. Am J Health Behav. 2007;31 Suppl 1:S36-46. doi:10.5555/ajhb.2007.31. supp.S36

20. Parker RM, Baker DW, Williams MV, Nurss JR. The test of functional health literacy in adults: a new instrument for measuring patients' literacy skills. J Gen Intern Med. 1995;10(10):537-541.

21. Agency for Healthcare Research and Qual (AHRQ). Medical Expenditure Panel Survey. http://meps.ahrq.gov/mepsweb/. Accessed December 1, 2011. Published 2011.

22. Vernon JA, Trujillo A, Rosenbaum S, DeBuono B. Low Health Literacy: Implications for National Health Care Policy. Washington, DC: George Washington University School of Public Health and Health Services; 2007.

23. Medical Expenditure Panel Survey (MEPS). MEPS topics: Prescribed drug use/events and expenditures. http://meps. ahrq.gov/mepsweb/data_stats/MEPS_topics.jsp?topicid=46Z-1. Accessed December 26, 2012. Published 2009.

24. Medical Expenditure Panel Survey (MEPS). MEPS HC-118A: 2008 Prescribed Medicines. Rockville, MD: Center for Financing, Access, and Cost Trends; 2010.

25. Martin LT, Ruder T, Escarce JJ, et al. Developing predictive models of health literacy. J Gen Intern Med. 2009;24(11):1211- 
1216. doi:10.1007/s11606-009-1105-7

26. Statistics USBoL. CPI inflation calculator. http://www.bls.gov/ data/inflation_calculator.htm. Accessed January 1, 2013. Published 2013.

27. Terza JV, Basu A, Rathouz PJ. Two-stage residual inclusion estimation: addressing endogeneity in health econometric modeling. J Health Econ. 2008;27(3):531-543. doi:10.1016/j. jhealeco.2007.09.009

28. Cameron AC, Trivedi PK. Regression Analysis of Count Data. Cambridge, UK: Cambridge University Press; 1998.

29. Mackun P, Wilson S. Population Distribution and Change: 20002010. Washington, DC: US Census Bureau; 2011.

30. Chen AY, Escarce JJ. Effects of family structure on children's use of ambulatory visits and prescription medications. Health Serv Res. 2006;41(5):1895-1914. doi:10.1111/j.14756773.2006.00584.x

31. Pylypchuk Y, Sarpong EM. Comparison of health care utilization: United States versus Canada. Health Serv Res. 2013;48(2 Pt 1):560-581. doi:10.1111/j.1475-6773.2012.01466.x

32. Friedland RB, O'Neill G. Understanding Health Literacy: New Estimates of the High Costs of Inadequate Health Literacy. Washington, DC: US Government Printing Office; 1998:57-91.

33. Wolf MS, Curtis LM, Waite K, et al. Helping patients simplify and safely use complex prescription regimens. Arch Intern Med. 2011;171(4):300-305. doi:10.1001/archinternmed.2011.39

34. Davis TC, Wolf MS, Bass PF 3rd, et al. Literacy and misunderstanding prescription drug labels. Ann Intern Med. 2006;145(12):887-894

35. Noureldin M, Plake KS, Morrow DG, Tu W, Wu J, Murray MD. Effect of health literacy on drug adherence in patients with heart failure. Pharmacotherapy. 2012;32(9):819-826. doi:10.1002/ j.1875-9114.2012.01109.x

36. Murray MD, Tu W, Wu J, Morrow D, Smith F, Brater DC. Factors associated with exacerbation of heart failure include treatment adherence and health literacy skills. Clin Pharmacol Ther. 2009;85(6):651-658. doi:10.1038/clpt.2009.7

37. Murray MD, Young J, Hoke S, et al. Pharmacist intervention to improve medication adherence in heart failure: a randomized trial. Ann Intern Med. 2007;146(10):714-725.

38. Gazmararian J, Jacobson KL, Pan Y, Schmotzer B, Kripalani $\mathrm{S}$. Effect of a pharmacy-based health literacy intervention and patient characteristics on medication refill adherence in an urban health system. Ann Pharmacother. 2010;44(1):80-87. doi:10.1345/aph.1M328

39. Gazmararian JA, Beditz K, Pisano S, Carreon R. The development of a health literacy assessment tool for health plans. J Health Commun. 2010;15(suppl 2):93-101. doi:10.108 0/10810730.2010.499986

40. Mosher HJ, Lund BC, Kripalani S, Kaboli PJ. Association of health literacy with medication knowledge, adherence, and adverse drug events among elderly veterans. J Health Commun. 2012;17(suppl 3):241-251. doi:10.1080/10810730.2012.712611

41. Blake SC, McMorris K, Jacobson KL, Gazmararian JA, Kripalani S. A qualitative evaluation of a health literacy intervention to improve medication adherence for underserved pharmacy patients. J Health Care Poor Underserved. 2010;21(2):559-567. doi:10.1353/hpu.0.0283

42. Review of Findings on Chronic Disease Self-Management Program (CDSMP) Outcomes: Physical, Emotional \& HealthRelated Quality of Life,Healthcare Utilization and Costs. http:// patienteducation.stanford.edu/research/Review_Findings_ CDSMP_Outcomes1\%208\%2008.pdf. Published 2013.

43. Ahn S, Basu R, Smith ML, et al. The impact of chronic disease self-management programs: healthcare savings through a community-based intervention. BMC Public Health. 2013;13:1141. doi:10.1186/1471-2458-13-1141

44. Kripalani S, Jacobson K, Brown S MK, Rask K, Jacobson T. Development and Implementation of a Health Literacy Training Program for Medical Residents. Medical Education online. 2006;11(13):1-8

45. Mackert M, Ball J, Lopez N. Health literacy awareness training for healthcare workers: improving knowledge and intentions to use clear communication techniques. Patient Educ Couns. 2011;85(3):e225-e228. doi:10.1016/j.pec.2011.02.022

46. Bowskill D, Garner L. Medicines non-adherence: adult literacy and implications for practice. Br J Nurs. 2012;21(19):1156-1159. 